\title{
Vida Chile 1998-2006: resultados y desafíos de la política de promoción de la salud en Chile
}

\author{
Judith Salinas, ${ }^{1}$ Anselmo Cancino, ${ }^{1}$ Sergio Pezoa, ${ }^{1}$ \\ Fernando Salamanca ${ }^{1}$ y Marina Soto ${ }^{1}$
}

Forma de citar

Salinas J, Cancino A, Pezoa S, Salamanca F, Soto M. Vida Chile 1998-2006: resultados y desafíos de la política de promoción de la salud en Chile. Rev Panam Salud Publica. 2007;21(2/3):136-44.

RESUMEN La prioridad politica otorgada por el Gobierno de Chile a la promoción de la salud se expresa en el desarrollo alcanzado por el Consejo Vida Chile y su Plan Nacional de Promoción de la Salud (PNPS). En este artículo se presenta el modelo evaluativo del PNPS chileno y sus principales resultados preliminares en el ámbito técnico y económico en el período 1998-2006. Para medir los resultados de las metas de proceso se utilizaron indicadores de cobertura (número de personas, de espacios promotores de la salud y de actividades) y del grado de cumplimiento de las estrategias. El número de actividades durante el período aumentó significativamente; $98 \%$ de los municipios del país cuentan con un plan comunal de promoción y un comité Vida Chile, que surge como instancia de trabajo intersectorial y ejecutora del Plan Comunal de Promoción; se incrementó el número de organizaciones sociales incorporadas a las estrategias de promoción de la salud; $34 \%$ de los establecimientos de educación del país (preescolar, básica y media) resultaron acreditadas como entidades promotoras de la salud; y aproximadamente $20 \%$ de la población total del país de todos los grupos de edad recibió beneficios directos de los planes comunales de promoción en 2006, entre otros logros. El costo total anual per capita de los planes comunales ascendió a \$US 6,60. Los principales factores facilitadores identificados fueron contar con la participación de las organizaciones sociales y redes comunitarias y disponer de un presupuesto adecuado para el plan comunal. Los factores obstaculizadores más frecuentes fueron la falta de tiempo o de recursos humanos para la promoción, la dispersión geográfica de la población y los problemas de accesibilidad a las actividades. Se hacen recomendaciones para la aplicación y el mejoramiento de los programas de promoción de la salud en la comunidad.

Palabras clave Promoción de salud, política de salud, evaluación de programas y proyectos de salud, Chile.

Departamento de Promoción de Salud y Participación Ciudadana, Ministerio de Salud, Santiago, Chile. La correspondencia se debe dirigir a Judith Salinas, Departamento de Promoción de Salud y Participación Ciudadana, Ministerio de Salud, Mac Iver No. 459, piso 7, Santiago, Chile. Correo electrónico: jsalinas@minsal.cl
En 1998, el Ministerio de Salud de Chile puso en marcha el Plan Nacional de Promoción de la Salud (PNPS), basado en la estrategia de desarrollar la atención primaria de salud y con el propósito de contribuir a enfrentar los desafíos del nuevo perfil epidemiológico social del país $(1,2)$. Más tarde se creó el Consejo Nacional para la Promoción de la Salud Vida Chile (refe- 
rida en lo adelante como Vida Chile), instancia de coordinación intersectorial presidida por el Ministro de Salud e integrada por 28 instituciones públicas y privadas de todo el país. Su función es asesorar a los ministerios para la elaboración de políticas saludables y coordinar un plan de acción estratégico de carácter intersectorial. Este Consejo cuenta con una red de consejos locales articulados bajo una idea motriz denominada Construyendo un País más Saludable. Los consejos Vida Chile comunales tienen un carácter participativo y están constituidos por representantes de organizaciones sociales y comunitarias, autoridades municipales, servicios públicos locales y entidades privadas. Sus objetivos básicos son colaborar con el diagnóstico participativo de la salud y la calidad de vida y participar en la formulación, implementación y evaluación del Plan Comunal de Promoción de Salud.

En el año 2000, a partir de una línea base establecida con los resultados de la Encuesta Nacional de Calidad de Vida y Salud (3-5), Vida Chile estableció cinco prioridades para esta década, con metas intersectoriales destinadas a reducir la prevalencia de obesidad, sedentarismo y tabaquismo y aumentar los factores protectores psicosociales y ambientales. La mayoría de estas metas pasaron a integrar los Objetivos Sanitarios de la Reforma de Salud (6). Para alcanzar estos objetivos, el Ministerio de Salud ha implementado de forma sistemática en todas las regiones del país los planes de promoción de la salud, consistentes en intervenciones comunitarias a nivel local y acciones de apoyo nacional para el desarrollo de los recursos humanos, la comunicación, la participación social, la reorientación de los servicios de salud y la regulación (7-10).

En una primera fase, las intervenciones locales dieron prioridad al trabajo con las organizaciones sociales (11), los establecimientos de educación (12) y la atención primaria de salud y, más tarde, a los centros de trabajo (13), las universidades (14) y las entidades del sector empresarial privado. Entre los hitos principales de este proceso están las tres ediciones del Congreso Chi- leno de Promoción de la Salud, celebrados en los años $1999,{ }^{2} 2002^{3}$ y enero 2007,4 y el Foro de las Américas, organizado por la Organización Panamericana de la Salud (OPS) en 2002, donde se suscribió el Compromiso de Chile para la Promoción de la Salud. En esos eventos se profundizó en el análisis político conceptual y en el conocimiento de las experiencias, se incentivó el proceso de evaluación y el uso de evidencias y se fortalecieron los vínculos y compromisos entre los diversos sectores de la sociedad chilena para construir un país con una mejor calidad de vida.

A partir de ese análisis, se implementaron de forma gradual y sistemática diferentes estrategias y programas que componen el PNPS, como la estrategia para declarar promotores de la salud a las escuelas, comunas y lugares de trabajo que cumplan con determinados requisitos; los programas para establecer ambientes libres de humo de tabaco (15); y la introducción de la actividad física en la atención primaria (16). Además, se estableció el Programa Salud con la Gente, consistente en otorgar reconocimiento $y$ apoyo al fortalecimiento de las organizaciones sociales vinculadas a la salud, al mejoramiento de la calidad de vida (autocuidado, adultos mayores, mujeres, vecinales, ambientales, juveniles y otras) y a la promoción de su incorporación a las instancias de participación social (11). A todo esto se sumaron campañas de comunicación social, realizadas durante un mes cada año para promover hábitos sanos de vida en la población general y en escolares (alimentación saludable, vida activa y ambiente libre del humo de tabaco).

\footnotetext{
Ministerio de Salud, Consejo VIDA CHILE, Organización Panamericana de la Salud. Libro de resúmenes I Congreso Chileno de Promoción de la Salud; 24 al 27 de agosto 1999; Santiago, Chile. Santiago: MINSAL; 1999.

3 Ministerio de Salud, Consejo VIDA CHILE, Organización Panamericana de la Salud. Libro de resúmenes II Congreso Chileno de Promoción de la Salud; 24 al 26 de octubre 2002; Santiago, Chile. Santiago: MINSAL; 2002.

4 Ministerio de Salud, Consejo VIDA CHILE, Organización Panamericana de la Salud. Libro de resúmenes III Congreso Chileno de Promoción de la Salud; 10 al 12 de enero 2007; Santiago, Chile. Santiago: MINSAL; 2007.
}

Estas campañas utilizaron medios locales de comunicación (afiches, volantes, gigantografías y cuñas radiales) según las guías técnicas elaboradas por Vida Chile, que contienen los 17 mensajes educativos validados para la población chilena $(17,18)$.

La promoción de la salud se entiende como un proceso mediante el cual se crean capacidades para que los individuos y las comunidades ejerzan un mayor control sobre los factores determinantes de su salud para poder mejorarla. El fundamento de este concepto es que el bien social se construye con la ciudadanía y todos los sectores de la sociedad y para ello se requiere, entre otras condiciones, voluntad política, visión común de largo plazo y respaldo financiero.

El Presidente de la República, Sr. Ricardo Lagos, al presentar las metas de salud hasta el año 2010 expresó ante el Consejo Vida Chile que "la forma más segura de alcanzar la equidad en salud es evitando la enfermedad, poniendo al alcance de todos los conocimientos e instrumentos que permitan mantener sana a la población. Por eso, nuestro compromiso es reforzar la salud pública de modo de actuar integralmente en la promoción de la salud y la prevención de la enfermedad". Más adelante, la aprobación de los cuerpos legales de la reforma de salud en 2004 (19) y la posterior participación de Chile como país promotor en la Comisión de Determinantes de la Salud de la OPS/OMS (20) agregaron nuevos espacios para el desarrollo de la política de promoción de la salud ${ }^{5}$ (21).

La voluntad política de las autoridades chilenas quedó reiterada por la Presidenta de la República, Dra. Michelle Bachelet, al incluir entre sus prioridades programáticas del sector salud en el período 2006-2010 la meta de "establecer con vigor la promoción de la salud en las políticas de atención primaria de salud", en un contexto en

\footnotetext{
Salinas J, Castanedo I, Harrison D, Le Vu A. The whole of government approach to promoting health. Case studies from Chile, Cuba, United Kingdom and Viet Nam [presentación oral]. 6th Global Conference on Health Promotion; 7-11 August 2005; Bangkok, Thailand
} 
el que el énfasis de la política social se basa en el desafío de otorgar mayor protección social, bienestar con equidad y participación ciudadana.

Con este compromiso público, la visión a largo plazo dada por la reforma de salud y sus metas hasta el año 2010, el impulso a la participación social y el aporte financiero sistemático del sector salud, el país enfrenta el gran desafío de construir la promoción de la salud como política del Estado.

En este artículo se presenta el modelo evaluativo del PNPS chileno y sus principales resultados preliminares en el ámbito técnico y económico en el período 1998-2006.

\section{MODELO EVALUATIVO DEL PLAN NACIONAL DE PROMOCIÓN DE LA SALUD}

El modelo de evaluación se diseñó con el objetivo de servir a los planes comunitarios y a las estrategias de promoción de la salud y se elaboró en forma multidisciplinaria con el aporte de las instancias locales, de expertos y de la literatura científica $(21,22)$. El modelo incorpora las dimensiones cualitativa y cuantitativa y se puede utilizar en forma total o parcial.

La evaluación se basa en la medición de las estructuras, los procesos, los resultados y el impacto de los programas, campañas y acciones realizados. En el trienio 1998-2000, el foco de la evaluación fue la medición de los indicadores de estructura y de procesos en tres ámbitos de evaluación: el políticoestratégico (posicionamiento, compromiso intersectorial y participación social), el de gestión (administración en los procesos para la eficiencia y eficacia de los planes locales) y el técnico (adecuación y calidad de las intervenciones). Más tarde, para la evaluación en el período 2001-2006, se incorporó la evaluación de los resultados y del impacto de las tareas realizadas. Para la evaluación de los resultados se construyó una línea base que permitió formular metas regionales y comparar los indicadores de calidad de vida de la población.

Como parte del proceso evaluativo, se realizó un análisis económico de sus resultados y una revisión sistemática para clasificar la evidencia en promoción (23). Esto permitió:

- identificar un conjunto de intervenciones comunitarias de promoción de la salud que permitieran estimar el monto de las inversiones presupuestarias en salud que el país pudiera mantener y que tuvieran un demostrado efecto en la disminución de la obesidad, el sedentarismo y el tabaquismo y en el desarrollo de los factores protectores psicosociales; y

- seleccionar intervenciones más efectivas y coherentes de acuerdo con el contexto sociocultural de la población chilena.

A continuación se describen los componentes del modelo evaluativo de la promoción:

\section{Construcción de la línea base}

Para construir una línea base que permitiera medir tanto los cambios en los estilos de vida y entornos de la población como el avance con respecto a las metas comprometidas, el Ministerio de Salud, en convenio con el Instituto Nacional de Estadísticas, realizó la Primera Encuesta Nacional de Calidad de Vida y Salud (ENCV) a fines del año 2000 (3-5). Además, se programó su aplicación cada cuatro años para medir el impacto de las políticas, las estrategias y los programas implementados y para proporcionar la información validada necesaria para hacer los ajustes pertinentes. La segunda ENCV se realizó en 2006 y se encuentra en fase de análisis.

La ENCV se aplica a una muestra de 6228 hogares, representativa de la población mayor de 15 años de zonas urbanas y rurales, tanto a nivel nacional como regional. Su objetivo es conocer la percepción de la población sobre su grado de satisfacción y sus estilos de vida, la prevalencia de factores condicionantes (especialmente de los factores priorizados en el PNPS: la alimentación, la actividad física, el consumo de tabaco, los factores psicosociales y ambientales), la prevalencia de las principales enfermedades crónicas y agudas y de accidentes, así como la prevalencia y el grado de discapacidad y las limitaciones de las personas para realizar las actividades de la vida cotidiana. Las cifras de prevalencia de los factores de riesgo obtenidas mediante la Encuesta Nacional de Salud en 2003 (24) confirmaron los resultados obtenidos en la primera ENCV del año 2000. Además, se aplicó la Encuesta Mundial de Tabaquismo en Jóvenes a una muestra de la población menor de 15 años en 2000 y $2003(25,26)$ y la Encuesta Mundial de Salud Escolar, en 2004. Dada la prioridad otorgada al tema del tabaquismo, se elaboró una línea base nacional específica para el programa Ambiente Libres del Humo de Tabaco, con la participación de los equipos de salud y los educadores $(27,28)$

Aunque estas encuestas son la fuente principal de información para la construcción de la línea base, también se tomó en cuenta la información sobre estilos de vida obtenida mediante otras encuestas periódicas realizadas por diferentes organismos públicos (Encuesta de Caracterización Socioeconómica Nacional, del Ministerio de Planificación y Cooperación, y las Encuestas sobre el Consumo de Drogas, realizadas por la Comisión Nacional de Control de Estupefacientes). También se utilizó la información proveniente de los sistemas de vigilancia sanitaria y alimentario-nutricional y los indicadores sociales y de salud que permiten realizar un análisis según el género y el grado de equidad (29). De esta forma, el PNPS cuenta con una línea base representativa de todo el país y de cada una de las trece regiones.

\section{Monitoreo y evaluación de los planes comunales de promoción}

Este proceso se realizó mediante pautas semestrales de monitoreo técnicofinanciero y pautas de planificación y evaluación anual. El primer ciclo de elaboración de Planes Regionales de Promoción de Salud fue trienal, de 1998 a 2000 (planes aprobados me- 
diante la resolución del Ministerio de Salud No. 817, del 14 de abril de 1999), y el segundo, sexenal, de 2001 a 2006 (Planes aprobados mediante la resolución No. 663 del 16 de abril de 2001).

Los propios equipos locales de promoción son los encargados de aplicar estos instrumentos de monitoreo y evaluación comunitarios. El Ministerio de Salud procesa y analiza la información, confecciona bases de datos, retroalimenta el sistema y propone medidas para mejorar la implementación y apoyar la toma de decisiones técnicas y financieras. Mediante el monitoreo y la evaluación se pueden identificar los equipos responsables, las instituciones y organizaciones participantes, los objetivos y las metas locales y su grado de cumplimiento, los integrantes y el funcionamiento de los Consejos Vida Chile comunales, las estrategias y actividades realizadas, las coberturas programadas y ejecutadas, los factores facilitadores y obstaculizadores, el financiamiento programado y ejecutado, los logros alcanzados y las recomendaciones para el período siguiente.

\section{Acreditación de espacios saludables}

El diseño de la metodología de acreditación de establecimientos de educación estuvo a cargo de la Comisión Salud-Educación de Vida Chile - compuesta por los ministerios de Educación, de Salud, la Junta Nacional de Auxilio Escolar y Becas, la Junta Nacional de Jardines Infantiles y la Fundación INTEGRA- y se implementó en forma descentralizada por los Consejos Vida Chile comunales (12). Esta metodología consta de procedimientos e instrumentos técnicos validados localmente, que buscan cumplir 10 condiciones y requisitos mínimos relacionados con la programación, la participación de la comunidad educativa -compuesta por los profesores, alumnos, padres, directivos y administrativos de un establecimiento- y la realización de actividades de promoción relacionadas con la alimentación, la actividad física, los aspectos psicosociales, el consumo de tabaco, alcohol y drogas y otros com- ponentes de la salud escolar. La acreditación de un establecimiento como Promotor de la Salud por parte de los ministros de Educación y de Salud depende del cumplimiento de estos requisitos y condiciones. Este proceso evaluativo, cuyos ejes son el incentivo y la retroalimentación para otros procesos innovadores y el mejoramiento de la gestión educativa continuada, ofrece importantes enseñanzas y contribuye a lograr el objetivo de contar con escuelas saludables.

A comienzos de 2003, el Consejo Vida Chile inició un proceso similar dirigido a formular metodologías que permitan acreditar lugares de trabajo como saludables, mediante un proceso participativo con representantes de los sectores laborales, sanitarios y académicos e instituciones laborales privadas. Este sistema comenzó a operar en 2006 - después de dos años de diseño y validación - con la evaluación de los primeros 120 lugares de trabajo, de los cuales se acreditaron 49. En 2006 también se comenzó a aplicar esta metodología en las universidades e instituciones de educación superior.

Por su parte, el sistema de acreditación y certificación de Ambientes Libres del Humo de Tabaco comenzó a aplicarse desde el año 2000 a los establecimientos de educación, salud, centros de trabajo y otros lugares públicos. A comienzos de 2006 se había alcanzado una cobertura de $85 \%$ en el sector salud, con acciones de educación y participación social para promover el control del tabaquismo; este proceso transcurrió en forma paralela a la preparación y discusión parlamentaria de la nueva Ley del Tabaco, la que fue aprobada finalmente en mayo 2006 (30).

\section{Sistematización de buenas prácticas locales}

Hasta el momento se han realizado tres procesos de sistematización y recopilación de información acerca de las buenas prácticas en la promoción de la salud, basado en guías metodológicas (31) elaboradas por el MINSAL. En el año 2000 se realizó el primer pro- ceso de sistematización de las experiencias locales que llevaran al menos dos años de ejecución. Para ello se contó con el análisis de la visión de los actores, sus testimonios, el análisis de las metodologías utilizadas y los logros alcanzados por 20 de las cerca de 1500 experiencias que estaban en curso (32). En 2001 se realizó una segunda sistematización de las intervenciones en una muestra de 126 establecimientos de educación promotores de la salud de diferentes regiones del país y de los niveles de educación preescolar, básico y medio. ${ }^{6}$ La tercera sistematización se llevó a cabo en 2005 en cinco lugares de trabajo: tres empresas privadas del sector productivo y financiero y dos de servicios públicos (33).

Estos procesos han aportado información útil para la toma de decisiones y el diseño de políticas y han contribuido a orientar las acciones y el papel que deben desempeñar las instituciones de Vida Chile. Además, la metodología empleada permitió identificar las instituciones líderes y las experiencias que deben extenderse, lo que constituye una importante fuente de aprendizaje y retroalimentación de los procesos locales.

\section{RESULTADOS DEL PLAN NACIONAL DE PROMOCIÓN DE LA SALUD}

A continuación se presentan los principales resultados derivados de una evaluación preliminar de la aplicación de la estrategia del PNPS en Chile. ${ }^{7}$

Para medir los resultados de las metas de proceso se utilizaron los indicadores de cobertura (número de personas, de espacios promotores de la

\footnotetext{
Jadue L, Ubilla S, Chomali M, Muñoz F. Sistematización de modelos de intervención en promoción de salud en establecimientos de educación. En: Ministerio de Salud, Consejo VIDA CHILE, Organización Panamericana de la Salud. Libro de resúmenes II Congreso Chileno de Promoción de la Salud; 24 al 26 de octubre 2002; Santiago, Chile. Santiago: MINSAL; 2002. Pp. 52.

7 La información que se ofrece a continuación solo tiene un carácter descriptivo; una vez concluido el análisis de la ENCV se presentarán los resultados definitivos para su publicación.
} 
CUADRO 1. Indicadores de proceso del Plan Nacional de Promoción de la Salud, Chile, 2001 y 2006

\begin{tabular}{lrr}
\hline \multicolumn{1}{c}{ Indicadores } & 2001 & 2006 \\
\hline $\begin{array}{l}\text { No. de beneficiarios directos } \\
\text { No. de comunas con Plan Comunal de Promoción } \\
\text { y Comité Vida Chile activos }\end{array}$ & 1804681 & 4251411 \\
$\begin{array}{l}\text { No. de organizaciones sociales en el Programa Salud } \\
\text { con la Gente }\end{array}$ & 307 & 342 \\
$\begin{array}{l}\text { No. de ambientes libres del humo de tabaco acreditados } \\
\text { No. de establecimientos de educación acreditados como } \\
\text { promotores de la salud }\end{array}$ & 1537 & 4547 \\
$\begin{array}{l}\text { No. de lugares de trabajo en proceso de acreditación } \\
\text { No. de plazas Vida Chile }\end{array}$ & 359 & 4063 \\
\hline
\end{tabular}

a La acreditación de lugares de trabajo se inició en el año 2006.

salud y de actividades) y del grado de cumplimiento de las estrategias, obtenidos mediante los sistemas de monitoreo de los planes comunales de promoción de todo el país (cuyo consolidado da cuenta de todas las acciones) y del sistema de acreditación intersectorial de espacios saludables. En términos generales se constataron importantes incrementos en la cobertura y el número de actividades durante esta etapa, con información anual para el período del 2001 al 2006. De los municipios del país, 342 (98\%) cuentan con un plan comunal de promoción y un comité Vida Chile que surge como instancia de trabajo intersectorial y ejecutora del Plan Comunal de Promoción.

El incremento en el número de organizaciones sociales incorporadas a las estrategias de promoción de la salud, establecimientos de educación promotores de la salud, ambientes libres de humo de tabaco y centros de trabajo —tanto de servicios públicos como empresas privadas - refleja el crecimiento de las intervenciones de promoción en el período 2001-2006 (cuadro 1).

En el caso de las instituciones educacionales, se han acreditado 4063 , es decir, cerca de $34 \%$ de los establecimientos de educación del país (preescolar, básica y media) ha incorporado acciones sistemáticas de promoción de la salud en coordinación con las autoridades educacionales y los gobiernos locales.

Se observó un incremento en el número de beneficiarios directos de los planes comunales de promoción en todos los grupos de edad, con un total de 4251411 personas en 2006 (aproximadamente $20 \%$ de la población total del país). Se constató también un aumento en la participación de la población escolar y adulta, con un incremento mayor en la población adolescente durante el último año (figura 1).

El nivel de financiamiento varió en dependencia de las diversas fuentes. El aporte financiero del sector salud - equivalente a US\$ 3,5 millones anuales (aproximadamente 1\% del presupuesto de la atención primaria)representó $51 \%$ del financiamiento intersectorial local (figura 2) y los recur-

\section{FIGURA 1. Beneficiarios del Plan Nacional de Promoción de la Salud según grupos de edad, Chile, 1999-2005}

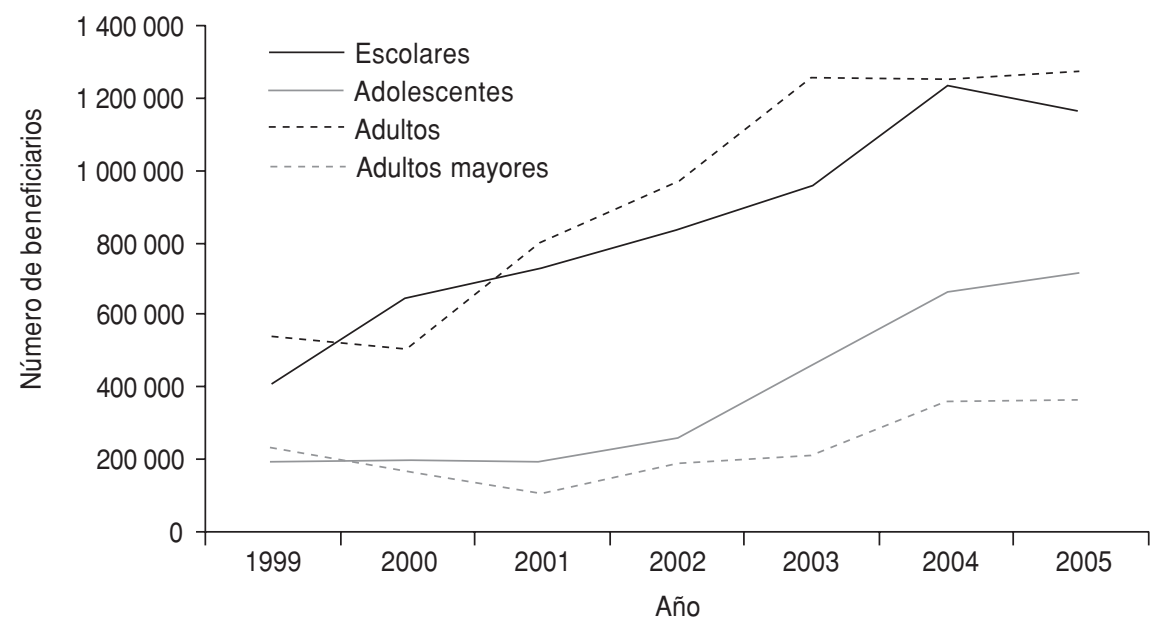

sos aportados por el Ministerio de Salud lograron tener un efecto movilizador. La disminución porcentual de los aportes financieros a niveles nacional y municipal en el último año se debe a la diferenciación introducida entre los aportes monetarios y no monetarios, a pesar que en las cifras municipales aún persisten aportes en infraestructura y equipos materiales. En cambio, los aportes comunitarios corresponden casi en su totalidad a aportes no monetarios, como el usufructo gratuito de locales y el trabajo voluntario.

Para analizar los resultados, los equipos de promoción toman en cuenta estas cifras, además de los resultados cualitativos logrados y los factores facilitadores y obstaculizadores encontrados en la gestión local de la promoción de la salud. Este análisis se realiza anualmente según las pautas mencionadas anteriormente que son autoaplicadas en cada comuna del país. En el cuadro 2 se presenta una síntesis de los factores facilitadores y obstaculizadores identificados al analizar los autoinformes sobre la gestión de 301 comunas en 2005. Se observó que los obstáculos más frecuentes son la falta de recursos humanos, los problemas de acceso físico a lugares donde se realizan actividades de promoción, el desinterés de otros sectores públicos y la falta de recursos económicos. Sin 
FIGURA 2. Fuentes de financiamiento del Plan Nacional de Promoción de la Salud, Chile, 1999-2005

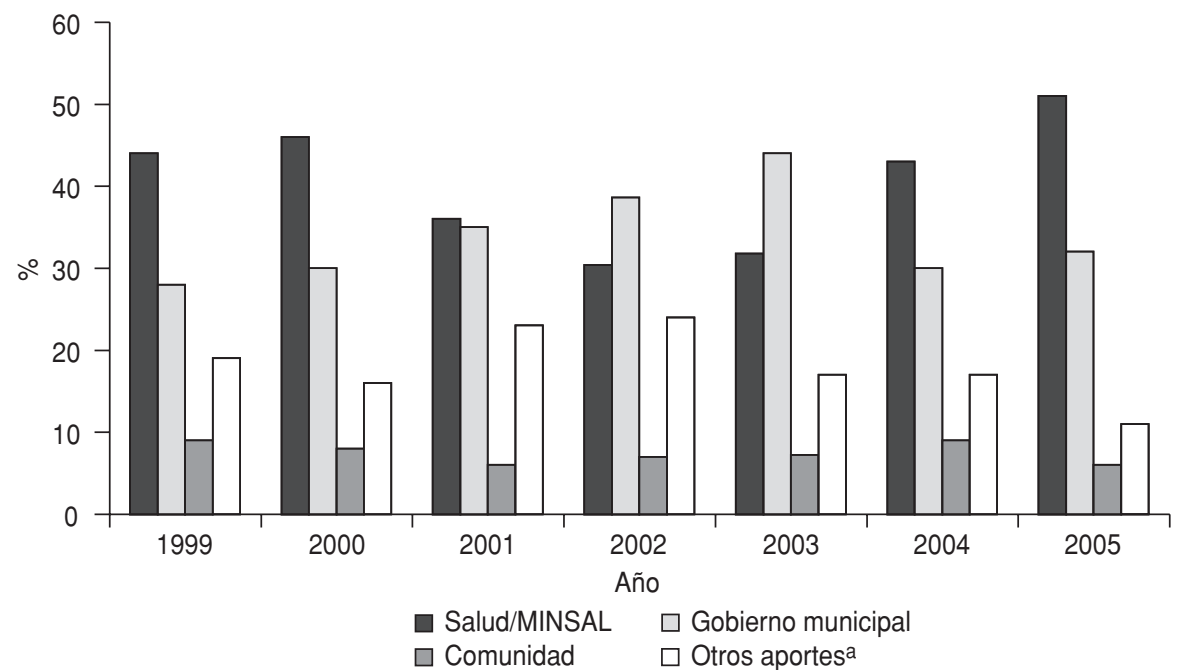

a En otros aportes se incluyeron los fondos de otros sectores públicos (educación, vivienda, trabajo, etc.) que apoyan los Planes Comunales de Promoción de la Salud.

embargo, cuando estos factores están resueltos se identifican frecuentemente como factores facilitadores. Los principales facilitadores fueron la participación activa de la comunidad, el trabajo coordinado con el sector educacional, la existencia de presupuesto adecuado y el apoyo de los departamentos municipales y sus autoridades edilicias.

En cuanto al costo de las intervenciones de salud realizadas, el costo total anual per capita de los planes co- munales ascendió a \$US 6,60 (cuadro 3). La estimación de los costos totales para cada prioridad del PNPS se obtuvo mediante la reconstitución de los costos del conjunto de actividades por actividad priorizada, cifra a la cual se agregaron las horas anuales de trabajo invertidas por el personal asalariado que trabajó en la intervención. Se observó una alta variabilidad en los costos: la intervención de menor costo fue la promoción en salud ambiental, probablemente por tener actividades más breves y recibir mayores aportes voluntarios. La segunda intervención de menor costo fue la campaña de ambientes libres del humo de tabaco, probablemente por su mayor grado de estandarización y la mayor dedicación de los equipos de salud. Las actividades relacionadas con los factores protectores psicosociales tuvieron un mayor costo relativo, ya que se basaron en actividades de mayor duración, requerían la presencia de profesionales y tuvieron menores aportes voluntarios. Las actividades más costosas por hora fueron las relacionadas con la promoción de la actividad física y de una alimentación sana debido a la

CUADRO 2. Factores facilitadores y obstaculizadores en la gestión de los Planes Comunales de Promoción de la Salud, ordenados según su incidencia, Chile, 2005a

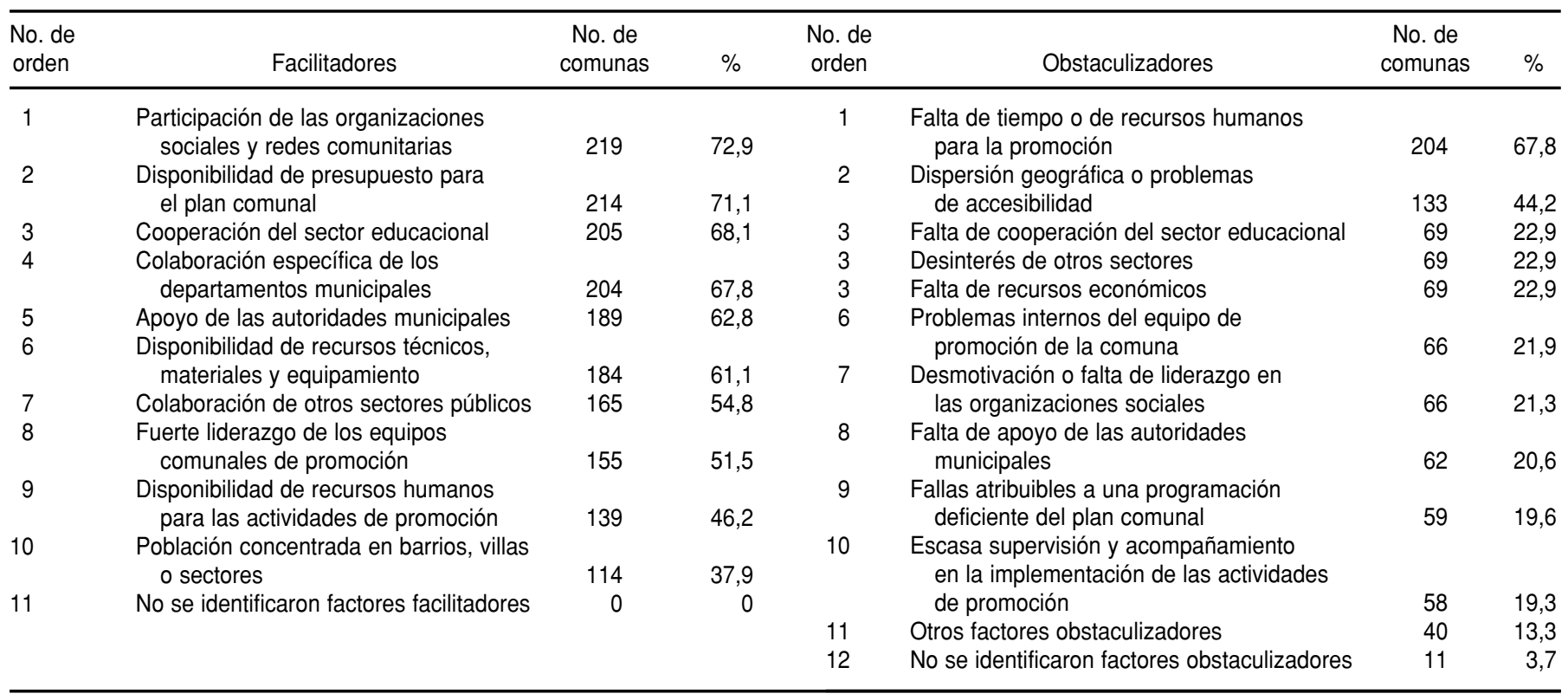

a Total de comunas: 301. 
CUADRO 3. Costo anual de las actividades del Plan Nacional de Promoción de la Salud, agrupadas según los factores condicionantes a que estaban dirigidas, Chile, $2000^{\mathrm{a}}$

\begin{tabular}{lc}
\hline \multicolumn{1}{c}{ Actividades } & $\begin{array}{c}\text { Costo anual } \\
\text { per cápita, US\$ }\end{array}$ \\
\hline Factores protectores & \\
ambientales & 1,25 \\
Tabaquismo & 2,11 \\
Factores protectores & \\
psicosociales & 4,10 \\
Alimentación & 5,92 \\
Actividad física & 9,59 \\
$\quad$ Promedio general & 6,57 \\
\hline a Datos preliminares. & \\
b El valor promedio del dólar americano durante el año 2000 \\
fue de 540,50 pesos chilenos, según la tasa de cambio ofi- \\
cial del Banco Central de Chile (http://www.bcentral.cl).
\end{tabular}

mayor inversión necesaria en recursos humanos y en equipamiento.

Según los resultados de la evaluación de dos proyectos pilotos contra la obesidad y a favor de la actividad física - uno con la población adulta de 40 consultorios de atención primaria y el otro con menores de tres centros educacionales- se logró reducir significativamente la tasas de obesidad y mejoró la percepción de los participantes acerca de su calidad de vida $(34,35)$.

Las principales dificultades enfrentadas hasta el momento estuvieron relacionadas con el predominio de una cultura sectorial basada en el paradigma biomédico, la resistencia administrativa a utilizar recursos públicos con organizaciones sociales, las tensiones entre diferentes concepciones de participación y promoción y la gran dificultad encontrada para trabajar en forma intersectorial. Además de la voluntad política de las autoridades superiores, las metas bien establecidas y los recursos suficientes, para alcanzar los objetivos trazados se requiere "permear" los distintos niveles de la administración pública y llegar con la prontitud requerida a los niveles locales con mensajes articulados entre los ministerios involucrados.

Las dificultades encontradas durante la implementación del proceso de monitoreo y evaluación estuvieron relacionadas con el déficit de recursos humanos, la rotación de los equipos y la falta de incentivos para la transferencia de aprendizajes. Por ello, para mantener este sistema de información periódica fue necesario simplificar las pautas e incluir un menor número de variables. En el futuro, este sistema de información debe formar parte de los sistemas de registros de salud pública, lo que permitiría, además, mantener un seguimiento del desarrollo de la promoción de salud en la red asistencial.

Respecto a las intervenciones, ha habido un déficit en el área de la comunicación social, ya que las campañas realizadas han sido breves y basadas en medios locales, sin la participación de los medios masivos de comunicación por lo costoso que resulta. En cuanto a la estrategia dirigida a declarar escuelas promotoras, se comienza a apreciar el agotamiento de ciertas actividades de vida saludable - mantenidas con el esfuerzo local y aportes del PNPS- por no ser parte del proyecto educativo ni contar con incentivos del Ministerio de Educación (por ejemplo la instalación de kioskos saludables y el aumento de horas de actividad física). Al mismo tiempo, se hizo necesario incorporar nuevas tecnologías informáticas en el proceso de acreditación e implementar un sistema electrónico de registros y certificaciones en línea, dado el volumen de información.

El establecimiento de metas para reducir la prevalencia de obesidad, tabaquismo y sedentarismo con vistas al año 2010 y la prioridad dada a cinco factores condicionantes (la alimentación, la actividad física, el consumo de tabaco, los factores protectores psicosociales y los ambientales) constituyeron un importante motor que imprimió una dirección clara al proceso de promoción de la salud en todo el país. Sin embargo, casi desde el principio se comenzó a percibir lo inalcanzable que resultaban y el riesgo de debilitar las acciones de empoderamiento y participación social, esencia de la promoción de salud. Por otra parte, los consejos Vida Chile comunales han definido nuevas prioridades locales (como la solución de problemas ambientales, la educación sexual y la lucha contra las drogas) que obligan a flexibilizar las metas y fortalecer las acciones comunitarias.

\section{CONCLUSIONES $Y$ RECOMENDACIONES}

El análisis preliminar del trabajo realizado durante nueve años por el PNPS y los consejos Vida Chile comunales demuestra que los resultados son altamente positivos, tanto en términos de los procesos construidos como en el posicionamiento del propio proyecto Vida Chile en la imagen pública y en su aceptación por los gobiernos regionales y locales. Además, se logró avanzar en el diseño y la instalación de intervenciones y estrategias intersectoriales, la formación de una masa crítica de gestores locales, la creación de redes y la organización de un movimiento social activo en torno a la construcción de un Chile más saludable y con mejor calidad de vida.

Desde el punto de vista del efecto de este programa en los cambios de comportamiento, se logró mejorar la percepción que tienen las personas acerca de sus hábitos y estilos de vida. Los cambios observados en el entorno y los espacios de la vida cotidiana son apreciados por las propias comunidades y por las instituciones locales y se expresa en la creciente adhesión de los sectores público y privado a iniciativas dirigidas a promover la salud.

Las limitaciones de la experiencia Vida Chile están relacionadas con la poca capacidad demostrada por el sector salud para alcanzar el compromiso efectivo de otros sectores, lo que reduce las posibilidades de lograr un impacto mayor. Por otra parte, si bien se ha logrado aumentar la cobertura, esta es aún insuficiente, ya que dado el perfil epidemiológico y social del país, se requiere invertir más recursos en la promoción de estilos de vida saludable. La experiencia del PNPS ha sido principalmente local y comunitaria, en los espacios de vida cotidiana de las personas, con menor desarrollo en los servicios de salud.

Esta experiencia demuestra que es posible llevar a cabo procesos evaluativos que apoyen al mejoramiento de planes y políticas y se basen en condiciones operacionales de funcionamiento del Ministerio de Salud, a pesar de los escasos recursos disponibles en la administración pública para evaluar programas. 
Esto tiene el valor agregado de retroalimentar el sistema y contribuye a genepara tomar decisiones. El bajo costo de estas acciones demuestra que con intervenciones de promoción adecuadas se puede ofrecer una mayor calidad de vida a las personas y a la comunidad.

Al diseñar procesos de evaluación de proyectos de promoción de la salud se deben tener presentes algunas consideraciones generales:

- No existen modelos específicos para la evaluación sistemática de proyectos de promoción de la salud, en parte porque sus resultados inciden en áreas de la salud pública que resultan muy complejas de evaluar, tienen menor desarrollo, están relacionadas con características psicosociales y culturales de las poblaciones y los procesos se basan en la participación ciudadana y la creación de capital social.

- Existen pocos estudios de medición de los resultados de este tipo de proyecto debido a que es difícil utilizar metodologías experimentales en el campo de las intervenciones sociales y, probablemente, por la necesidad de contar con un período largo de observación para poder ver los resultados.

- La falta de consenso acerca del tipo de evidencia necesaria para evaluar la promoción de la salud puede llerar la información científica necesaria

var a falsas conclusiones, que incluso pueden ser contraproducentes o restrictivas para las estrategias y procesos implementados. En el caso chileno, la opción ha sido considerar tanto la evidencia social y científica como el análisis político.

- Debido a que el abordaje poblacional de la promoción de la salud se realiza mediante estrategias integrales, múltiples y participativas, se deben usar metodologías de evaluación que tomen en cuenta la naturaleza de las intervenciones y el ciclo de planificación-evaluación establecido. En el PNPS se ha realizado un gran esfuerzo - aunque no siempre se ha logrado- para reflejar la complejidad de los fenómenos sociales y los cambios culturales planteados.

De acuerdo con la experiencia acumulada en el diseño y la utilización de los componentes del modelo evaluativo en Chile, se puede concluir que las propuestas formuladas deben estar dirigidas a:

- aumentar las capacidades de investigación y vigilancia sobre los determinantes sociales de la salud y sus factores protectores;

- fortalecer el sistema de seguimiento y evaluación de los planes y estrategias de promoción de la salud; y

- crear instancias de información, análisis, abogacía y comunicación social.

\section{REFERENCIAS}

1. Salinas J, Donoso N, Molina H, Montecinos C, Pezoa S, Torres M, eds. Plan Nacional de Promoción de la Salud. Santiago, Chile: Mosquito Comunicaciones; 1999.

2. Belmar R, Salinas J. Atención primaria y participación comunitaria en salud: orientaciones estratégicas. Rev Med Chile. 1994;122:215-20.

3. República de Chile, Ministerio de Salud. Encuesta de Calidad de Vida y Salud, Chile 2000. Santiago, Chile: MINSAL; 2003.

4. González C, Aguilera X, Betancur C, Guerrero $\mathrm{H}$, Salinas J, Donoso N. Encuesta de Calidad de Vida y Salud, MINSAL-INE 2000. El Vigía (Chile). 2002;5(16):2-8.

5. González C, Aguilera X, Betancur C, Guerrero A, Salinas J, Donoso N. Características demográficas y socioeconómicas de la población. Encuesta de Calidad de Vida y Salud, Chile 2000. El Vigía (Chile). 2003;6(17):2-6. Objetivos Sanitarios para la Década 20002010. Resumen ejecutivo. El Vigía (Chile). 2002; 5(15):2-11.

7. Salinas J, Vio F. Promoción de la salud en Chile. Rev Chil Nutr. 2002;29(S1):64-173.

8. Salinas J. Vida Chile 1998-2003: achievements and challenges of health promotion as a public policy. Health Targets: News \& Views. 2004;7(1):8-9.

9. Albala C, Olivares S, Salinas J, Vio F, eds. Bases, prioridades y desafíos de la promoción de la salud. Santiago, Chile: Andros Impresores; 2004.

10. Salinas J, Escribano I. Promoción de la salud y educación para la salud en Chile. En: Arroyo $\mathrm{H}$, ed. La promoción de la salud en América Latina: modelos, estructuras y visión crítica.
6. República de Chile, Ministerio de Salud. Los
Por último, las políticas nacionales de promoción de la salud deben enfrentar los siguientes desafíos:

- consolidar lo avanzado y fortalecer las acciones comunitarias para lograr un cambio cultural;

- fortalecer el compromiso de los gobiernos locales y regionales y de la sociedad civil con las metas del país para el año 2010;

- consolidar los acuerdos con los sectores de educación y trabajo y establecer nuevas alianzas estratégicas con el sector privado, los medios de comunicación y las asociaciones de municipios, entre otras;

- incorporar de manera transversal y eficaz la actividad de promoción de la salud en el modelo de atención sanitaria y como parte del quehacer de la salud pública; y

- avanzar decididamente en la formulación de políticas saludables y en el establecimiento de los marcos regulatorios necesarios.

En este sentido, la construcción de políticas públicas saludables, sumada a la consolidación del trabajo comunitario para desarrollar capacidades que permitan ejercer un control social sobre los factores determinantes de la salud, contribuirán a lograr un mayor bienestar y una mejor calidad de vida de la población.
San Juan, Puerto Rico: Universidad de Puerto Rico; 2004. Pp. 155-88.

11. República de Chile, Ministerio de Salud. Salud con la Gente. Programa de reconocimiento y apoyo a las organizaciones sociales de salud. Santiago, Chile: MINSAL; 1998.

12. República de Chile, Ministerio de Salud, Ministerio de Educación, OPS/OMS. Establecimientos de educación promotores de la salud en Chile: avances y desafíos. Santiago, Chile: Mosquito Comunicaciones; 2003.

13. Calderón B, Salinas J, Vio F, eds. Vida sana en la empresa: guía practica para empresas. Santiago, Chile: Acción RSE, INTA, MINSAL; 2004.

14. Lange I, Vio F. Guía para universidades saludables y otras instituciones de educación superior. Santiago, Chile: Andros Impresores; 2006. 
15. Bello S, Soto M, Salinas J. Salud sin tabaco: guía técnica metodológica. Programa Ambientes Libres del Humo de Tabaco. Santiago, Chile: Impreso La Nación; 2001.

16. Salinas J, Vio F. Promoción de la salud y actividad física: una política prioritaria. Rev Panam Salud Publica. 2003;14(4):28-8.

17. República de Chile, Ministerio de Salud. Guía educativa para una vida saludable: guías alimentarias, actividad física y tabaco. Santiago, Chile: MINSAL; 2005. (Resol. Ex. No. 459 del 10 de agosto de 2005).

18. Vio F, Salinas J, eds. Guía para una vida activa. Santiago, Chile: Andros Impresores; 2003.

19. República de Chile, Ministerio de Salud. Ley de Autoridad Sanitaria No. 19.937. Diario Oficial de Chile 200424 febrero.

20. Faúndez A, ed. Construyendo la nueva agenda social desde la mirada de los determinantes sociales de la salud. Documento síntesis del ciclo de diálogos democráticos. Santiago, Chile: Facultad Latinoamericana de Ciencias Sociales, Ministerio de Salud, OPS/OMS; 2005.

21. European Commission, International Union for Health Promotion and Education. The evidence of health promotion effectiveness: shaping public health in a new Europe. Bruselas, Luxemburgo: Jouve Composition, Impresion Press; 1999.

22. Salinas J. Health promotion in Chile: an evaluation of a national plan implementation. Promotion and Education. 2000:4:13-6.
23. Valenzuela MT, Jadue L, Peña P, Vega J. Propuesta de acciones poblacionales de promoción de la salud en el plan de salud pública del sistema AUGE. Santiago, Chile: Iniciativa Chilena de Equidad en Salud; 2003.

24. República de Chile, Ministerio de Salud. Encuesta Nacional de Salud 2003. Resumen ejecutivo. El Vigía (Chile). 2004;8(20):1-20.

25. González C, Chiu M, Szot M, Aguilera X. Tendencias en el consumo de tabaco en escolares chilenos: resultados de la Encuesta Mundial de Tabaquismo en Jóvenes (EMTA). Regiones Metropolitana y Valparaíso 2000 y 2003. El Vigia (Chile). 2004;8(21):18-22.

26. Burgos A, González C, Chiu M, Szot M. Encuesta Mundial de Tabaquismo en Jóvenes 2003 (GYTSS 2003). El Vigía (Chile). 2004; 8(22):2-8.

27. Bello S, Soto M, Michalland S, Salinas J. Encuesta Nacional de Tabaquismo en Funcionarios de Salud. Rev Med Chile. 2004;132: 223-32.

28. Bello S, Michalland M, Soto M, Salinas J. Tabaquismo en funcionarios de educación. Rev Chil Enf Respir. 2004;20:131-8.

29. Salinas J. Estilos de vida. Hacia un país saludable. En: Jadue L, Marín F, eds. Determinantes sociales de la salud en Chile. En la perspectiva de la equidad. Santiago, Chile: Impresión Grafic Suisse; 2005. Pp. 81-6.

30. República de Chile, Ministerio de Salud. Ley No. 20.105 modifica Ley 19.419 en materias re- lativas a la publicidad y consumo de tabaco. Diario Oficial de Chile 200616 mayo.

31. Kahan B, Goodstadt M. The interactive domain model of best practices in health promotion: developing and implementing a best practices approach to health promotion. Health Promot Pract. 2001;2(1):43-67.

32. República de Chile, Ministerio de Salud. Promoción de salud y equidad. Hechos y testimonios. Santiago, Chile: MINSAL; 2000.

33. Salinas J, ed. Construyendo políticas públicas saludables en el lugar de trabajo: el aporte de cinco experiencias. Santiago, Chile: MINSAL; 2005.

34. Kain J, Vio F, Leyton D, Cerda R, Olivares S, Uauy $R$, et al. Estrategia de promoción de salud en escolares de educación básica municipalizada de la comuna de Casablanca, Chile. Rev Chil Nutr. 2005;32(2):126-32.

35. Salinas J, Bello M, Flores A, Carbullanca L, Torres M. Actividad física integral con adultos y adultos mayores: resultados de un programa piloto. Rev Chil Nutr. 2005;32(3):215-24.

Manuscrito recibido el 27 de septiembre de 2004. Aceptado para publicación, tras revisión, el 23 de febrero de 2007.
ABSTRACT

The Vida Chile program: results and challenges with health promotion policy in Chile, 1998-2006

\footnotetext{
Key words
}

The Government of Chile has placed a high priority on health promotion. This is evident in the advances made through its National Plan for Health Promotion (Plan Nacional de Promoción de la Salud) and the Vida Chile National Council for Health Promotion (Consejo Nacional para la Promoción de la Salud Vida Chile). Chaired by the minister of health, Vida Chile is made up of 28 public and private institutions from around the country. Vida Chile has a network of local councils that have been established in the country's comunas (communes, or local-level divisions of the country's provinces) and that include government officials and representatives of local societal and community organizations and private businesses. This report details the methods used to evaluate the National Plan as well as provides a preliminary assessment of the technical and financial results for the 1998-2006 period. Coverage indicators (number of participants; number of accredited health-promoting schools, workplaces, and universities; and number of health promotion events) and the extent of strategy implementation were used to measure the success of the program. Health promotion activities grew markedly during this period. Among the notable accomplishments were the following four: (1) $98 \%$ of the communes now have their own community health promotion plan and intersectoral Vida Chile committee to implement the plan, (2) there has been an increase in societal and community groups involved in the health promotion strategies, (3) 34\% of the primary and secondary schools have become accredited health-promoting schools, and (4) approximately $20 \%$ of the total population benefited directly from community-health-plan activities in 2006. The average per capita cost of the community health plans' activities in 2006 was US\$ 6.60. The two most important factors that facilitated the operation of the local health promotion plans were participation by community and societal groups and having an adequate budget for the local activities. Hindering factors included a lack of time and/or human resources to devote to health promotion, a geographically dispersed population, and difficulty in accessing the activities.

Health promotion, health policy, program evaluation, Chile. 